\title{
Least-squares finite element methods for the Navier-Stokes equations for generalized Newtonian fluids
}

\author{
Serdar Serdas $^{1 *}$, Alexander Schwarz ${ }^{1}$, Jörg Schröder ${ }^{1}$, Stefan Turek ${ }^{2}$, Abderrahim Ouazzi ${ }^{2}$, and Masoud \\ Nickaeen $^{2}$ \\ ${ }^{1}$ Institute of Mechanics, Faculty of Engineering, University of Duisburg-Essen, Universitätsstr. 15, 45117 Essen, Germany \\ 2 Institute for Applied Mathematics, Faculty of Mathematics, TU Dortmund University, Vogelpothsweg 87, 44227 \\ Dortmund, Germany
}

\begin{abstract}
In this contribution we present the least-squares finite element method (LSFEM) for the incompressible Navier-Stokes equations. In detail, we consider a non-Newtonian fluid flow, which is described by a power-law model, see [1]. The second-order problem is reformulated by introducing a first-order div-grad system consisting of the equilibrium condition, the incompressibility condition and the constitutive equation, which are written in residual forms, see [2]. Here, higher-order finite elements which are an important aspect regarding accuracy for the present formulation are investigated.
\end{abstract}

Copyright line will be provided by the publisher

\section{Introduction}

The classical LSFEMs provide some theoretical and computational advantages, see e.g. [3], but there are still difficulties concerning, e. g. the mass conservation, especially when lower-order interpolants are used, see [4]. Besides the application of some weighting factors, a possible solution is the consideration of higher interpolations, see e.g. [5]. In the present work, we compare quadratic and cubic formulations for non-Newtonian fluids by a numerical example.

\section{Least-squares method}

We consider the velocity-stress-pressure approach for the stationary non-Newtonian fluid equations which are given by the balance of momentum, mass conservation and the material equation as following

$$
\rho \nabla \boldsymbol{v} \boldsymbol{v}-\operatorname{div} \boldsymbol{\sigma}=\mathbf{f}, \quad \operatorname{div} \boldsymbol{v}=0 \quad \text { and } \quad \boldsymbol{\sigma}-2 \rho \nu\left(D_{I I}\right) \nabla^{s} \boldsymbol{v}+p \mathbf{I}=\mathbf{0}
$$

with some suitable boundary conditions. Here, $\boldsymbol{\sigma}$ denotes the Cauchy stresses, $\mathbf{f}$ the forcing function, $\boldsymbol{v}$ the velocities, $p$ the pressure, $\rho$ the density, and $\nu(\cdot)$ is the (nonlinear) viscosity. The symmetric part of the deformation rate tensor is defined as $\nabla^{s} \boldsymbol{v}=\frac{1}{2}\left(\nabla \boldsymbol{v}+[\nabla \boldsymbol{v}]^{T}\right)$ and the second invariant of the deformation rate tensor as $D_{I I}=\frac{1}{2}\left(2 \nabla^{s} \boldsymbol{v} \cdot 2 \nabla^{s} \boldsymbol{v}\right)$. Here, we chose for the viscosity function the power-law model to describe the non-Newtonian fluid behavior

$$
\nu\left(D_{I I}\right)=\nu_{0} D_{I I}^{\frac{n-1}{2}}, \quad\left(\nu_{0}>0\right)
$$

where $\nu_{0}$ is the zero shear rate viscosity and $n$ the flow behavior index which distinguishes between different type of fluids. For $n=1$, we recover the Newtonian fluid (constant viscosity). For $n>1$ one obtain shear-thickening (or dilatant) fluids (viscosity increases with increase in shear-rate) and for $n<1$ shear-thinning (or pseudoplastic) fluids (viscosity decreases with increase in shear-rate). Furthermore, we replace the nonlinearities such as the convective term and viscosity term by the Newton linearization technique. Using quadratic $L^{2}$-norms, the linearized physically weighted least-squares functional is constructed as

$$
\begin{aligned}
\mathcal{J}_{\text {lin }}\left(\boldsymbol{v}, \boldsymbol{\sigma}, p ; \boldsymbol{v}^{k}\right) & =\frac{1}{2}\left\|\frac{1}{\sqrt{\rho}}\left(\rho \nabla \boldsymbol{v}^{k} \boldsymbol{v}+\rho \nabla \boldsymbol{v} \boldsymbol{v}^{k}-\operatorname{div} \boldsymbol{\sigma}-\mathbf{f}\right)+\mathcal{Q}_{\text {conv }}^{k}\right\|_{0}^{2}+\frac{1}{2}\|\operatorname{div} \boldsymbol{v}\|_{0}^{2} \\
& +\frac{1}{2}\left\|\frac{1}{\sqrt{\rho \nu\left(D_{I I}\right)^{k}}}\left(\boldsymbol{\sigma}-2 \rho \nu\left(D_{I I}\right)^{k} \nabla^{s} \boldsymbol{v}-8 \rho \nu^{\prime}\left(D_{I I}\right)^{k}\left(\nabla^{s} \boldsymbol{v} \cdot \nabla^{s} \boldsymbol{v}^{k}\right) \nabla^{s} \boldsymbol{v}^{k}+p \mathbf{I}\right)+\mathcal{Q}_{v i s}^{k}\right\|_{0}^{2}
\end{aligned}
$$

where the index $k$ is taken as either an initial guess or as a known quantity from the immediate previous iteration. $\mathcal{Q}_{\text {conv }}^{k}$ and $\mathcal{Q}_{\text {vis }}^{k}$ are denoting terms from the linearization which are only related to known values. The minimization of $\mathcal{J}_{\text {lin }}$ requires the first variation $\delta \mathcal{J}_{l i n}$ to be equal to zero. We use mixed finite elements $R T_{m} P_{k} P_{l}$, where $P_{k}$ and $P_{l}$ denote Lagrange shape functions of polynomial order $k$ for the velocities and $l$ for the pressure. $R T_{m}$ denotes Raviart-Thomas interpolants of polynomial order $m$ for a conforming discretization of the stresses. Further remarks regarding the minimization of $\mathcal{J}_{\text {lin }}$ or the used finite element spaces are given in [3] and [6].

\footnotetext{
* Serdar Serdas: Email serdar.serdas@uni-due.de, phone: +00 49201 183-3792, fax: +00 49201 183-2680
} 


\section{Numerical example}

As a numerical example we solve a fully developed power law fluid flow between parallel plates. Figure 1 shows the flow domain and the boundary conditions. Due to the symmetry, we consider only the upper half of the domain.

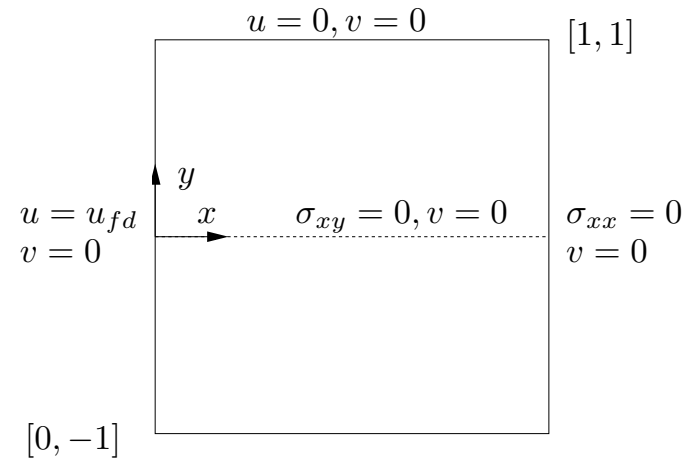

Fig. 1: Boundary value problem for a fully developed power law fluid flow between parallel plates.

For the inflow boundary condition, the horizontal velocity $u_{f d}=\frac{u}{u_{a v g}}$ is imposed by the analytical velocity profile (4) and the vertical velocity is set equal to zero. The upper edge has no-slip boundary conditions, the symmetry line a zero shear-stress $\sigma_{x y}$ and zero vertical velocity $v=0$. The outflow has a zero normal-stress boundary condition $\sigma_{x x}=0$ and a zero vertical velocity $v=0$. The material parameter such as the density $\rho$ and the flow consistency $\nu_{0}$, are set to one.

$$
u_{f d}=\frac{2 n+1}{n+1}\left(1-y^{\frac{n+1}{n}}\right), \quad y=[0,1]
$$
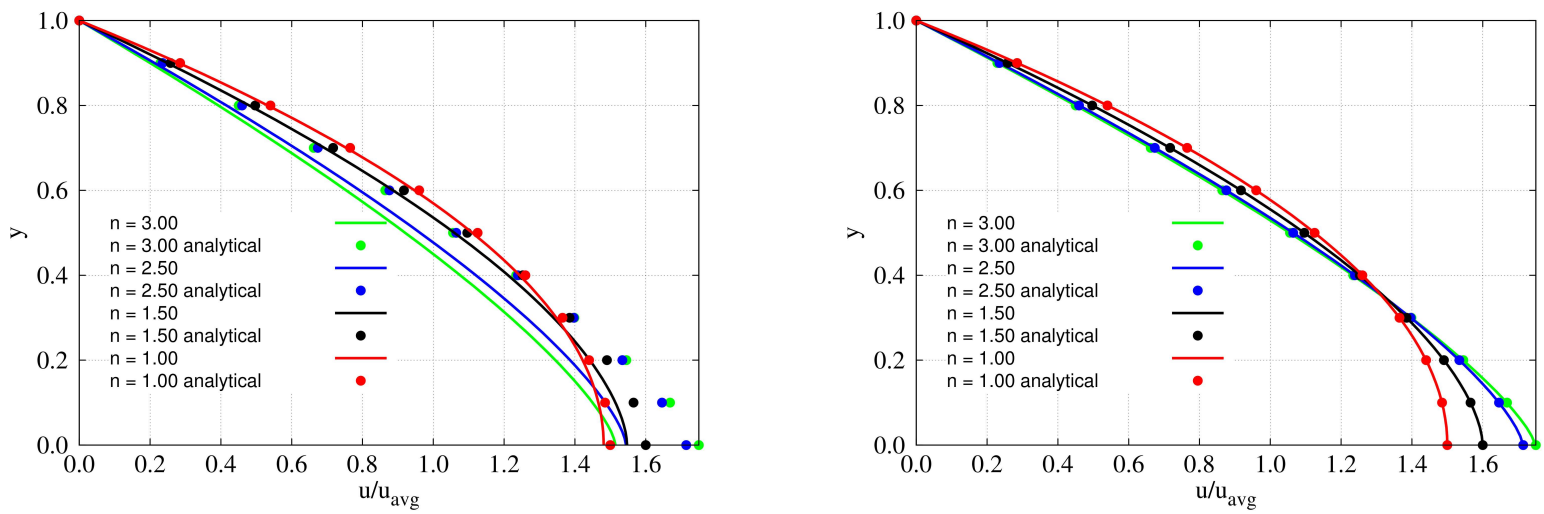

Fig. 2: Comparison of the velocity profiles with the analytical solution for various power law index values regarding shear-thickening fluids for $R T_{0} P_{2} P_{1}$ (left) and $R T_{1} P_{3} P_{1}$ (right) discretizations.

Figure 2 depicts various flow behavior index for shear-thickening fluids. On the left, the results for the $R T_{0} P_{2} P_{1}(62,339$ dofs) and on the right the $R T_{1} P_{3} P_{1}$ (40,643 dofs) discretizations can be seen. The results of the outflow velocities are compared with the analytical velocity profile (4). As could be expected, an increase of the parameter $n$ in (2) leads to a more steeper velocity profile. Considering the $R T_{0} P_{2} P_{1}$ discretization, it turns out that the lower-order discretization shows difficulties to predict the analytical solutions whereas the higher-order discretization matches very well with the analytical solution.

Acknowledgements MERCUR Pr-2011-0017: "Effiziente Simulationstechniken für robuste Least-Squares FEM in der Fluiddynamik"

\section{References}

[1] B.C. Bell, and K.S. Surana, IJNMF 18, 127-162 (1994).

[2] P.B. Bochev and M.D. Gunzburger, CMAME 126, 267-287 (1995).

[3] B.N. Jiang, The Least-Squares Finite Element Method. Springer-Verlag, (1998).

[4] S. Serdas, A. Schwarz, J. Schröder, S. Turek, A. Ouazzi and M. Nickaeen, PAMM 13, 301-302 (2013).

[5] M. Nickaeen, A. Ouazzi and S. Turek, JOCP 256, 416-427 (2014).

[6] A. Schwarz and J. Schröder, PAMM 11, 589-590 (2011). 